\title{
RESEARCH
}

Open Access

\section{Hypoxia-preconditioned mesenchymal stem cells prevent renal fibrosis and inflammation in ischemia-reperfusion rats}

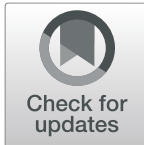

Naoki Ishiuchi ${ }^{1}$, Ayumu Nakashima ${ }^{1,2^{*}}$, Shigehiro Doi ${ }^{1}$, Ken Yoshida', Satoshi Maeda ${ }^{2,3}$, Ryo Kanai ${ }^{1}$, Yumi Yamada ${ }^{1}$, Takeshi Ike', Toshiki Doi ${ }^{1}$, Yukio Kato ${ }^{2,3}$ and Takao Masaki ${ }^{* *}$

\begin{abstract}
Background: Mesenchymal stem cells (MSCs) have been reported to promote the regeneration of injured tissue via their paracrine abilities, which are enhanced by hypoxic preconditioning. In this study, we examined the therapeutic efficacy of hypoxia-preconditioned MSCs on renal fibrosis and inflammation in rats with ischemia-reperfusion injury (IRI).

Methods: MSCs derived from rats and humans were incubated in $1 \% \mathrm{O}_{2}$ conditions ( $\left.1 \% \mathrm{O}_{2} \mathrm{MSCs}\right)$ for $24 \mathrm{~h}$. After IRI, $1 \% \mathrm{O}_{2}$ MSCs or MSCs cultured under normoxic conditions $\left(21 \% \mathrm{O}_{2}\right.$ MSCs) were injected through the abdominal aorta. At 7 or 21 days post-injection, the rats were sacrificed and their kidneys were analyzed. In in vitro experiments, we examined whether $1 \% \mathrm{O}_{2}$ MSCs enhanced the ability to produce anti-fibrotic humoral factors using transforming growth factor (TGF)- $\beta 1$-stimulated HK-2 cells incubated with conditioned medium from MSCs.
\end{abstract}

Results: Administration of rat $1 \% \mathrm{O}_{2}$ MSCs $\left(1 \% \mathrm{O}_{2}\right.$ rMSCs) attenuated renal fibrosis and inflammation more significantly than rat $21 \% \mathrm{O}_{2}$ MSCs. Notably, human $1 \% \mathrm{O}_{2}$ MSCs $\left(1 \% \mathrm{O}_{2}\right.$ hMSCs) also attenuated renal fibrosis to the same extent as $1 \% \mathrm{O}_{2}$ rMSCs. Flow cytometry showed that $1 \% \mathrm{O}_{2}$ hMSCs did not change human leukocyte antigen expression. Further in vitro experiments revealed that conditioned medium from $1 \% \mathrm{O}_{2}$ MSCs further suppressed TGF- $\beta 1$-induced fibrotic changes in HK-2 cells compared with $21 \% \mathrm{O}_{2}$ MSCs. Hypoxic preconditioning enhanced vascular endothelial growth factor (VEGF) and hepatocyte growth factor (HGF) secretion. Interestingly, VEGF knockdown in $1 \% \mathrm{O}_{2} \mathrm{MSC}$ attenuated HGF secretion and the inhibition of TGF- $\beta 1$-induced fibrotic changes in HK-2 cells. In addition, VEGF knockdown in $1 \% \mathrm{O}_{2}$ hMSCs reduced the anti-fibrotic effect in IRI rats.

Conclusions: Our results indicate that hypoxia-preconditioned MSCs are useful as an allogeneic transplantation cell therapy to prevent renal fibrosis and inflammation.

Keywords: Mesenchymal stem cells, Hypoxia, Vascular endothelial growth factor, Hepatocyte growth factor, Renal fibrosis

\footnotetext{
*Correspondence: ayumu@hiroshima-u.ac.jp; masakit@hiroshima-u.ac.jp 'Department of Nephrology, Hiroshima University Hospital, 1-2-3 Kasumi, Minami-ku, Hiroshima 734-8551, Japan

Full list of author information is available at the end of the article
}

(c) The Author(s). 2020 Open Access This article is licensed under a Creative Commons Attribution 4.0 International License, which permits use, sharing, adaptation, distribution and reproduction in any medium or format, as long as you give appropriate credit to the original author(s) and the source, provide a link to the Creative Commons licence, and indicate if changes were made. The images or other third party material in this article are included in the article's Creative Commons licence, unless indicated otherwise in a credit line to the material. If material is not included in the article's Creative Commons licence and your intended use is not permitted by statutory regulation or exceeds the permitted use, you will need to obtain permission directly from the copyright holder. To view a copy of this licence, visit http://creativecommons.org/licenses/by/4.0/. The Creative Commons Public Domain Dedication waiver (http://creativecommons.org/publicdomain/zero/1.0/) applies to the data made available in this article, unless otherwise stated in a credit line to the data. 


\section{Background}

The prevalence of chronic kidney disease (CKD) is estimated to be $10-15 \%$ worldwide $[1,2]$. Therefore, it is currently recognized as a world health concern with evidence that CKD patients have an increased risk of not only cardiovascular diseases, but also all-cause mortality $[3,4]$. In clinical settings, CKD occurs in the elderly and patients with chronic diseases that cause renal damage, such as chronic glomerular nephritis, hypertension, and diabetes mellitus [5-7]. On the other hand, acute kidney injury (AKI) is defined as an abrupt decrease in renal function, and most patients should recover to their baseline level after AKI. However, recent studies have revealed that a significant number of AKI patients eventually develop CKD [8-10], so called "AKI to CKD transition." Thus, AKI has attracted attention as a novel risk factor of CKD.

Pathologically, interstitial fibrosis and inflammation are common features in CKD patients regardless of primary disease $[11,12]$. Although ischemia-reperfusion injury (IRI) is a well-established rodent model of AKI, progression of renal fibrosis and inflammation are also observed in the kidney after IRI [13]. According to previous studies, several pathological mechanisms, such as hypoxia, microvascular rarefaction, inflammation, transforming growth factor (TGF)- $\beta 1$ production, and epithelial-mesenchymal transition (EMT), reportedly participate in AKI to CKD transition [14-16]. However, there are currently no effective therapies to prevent AKI to CKD progression.

Some studies have reported that the administration of mesenchymal stem cells (MSCs) exerts beneficial effects against various diseases such as heart disease [17], stroke [18], and autoimmune disease [19]. MSCs were previously considered to promote the regeneration of injured tissue by their capacity to differentiate into multiple tissue types $[20,21]$. However, rather than differentiating, paracrine activities are likely implicated in the therapeutic effects of MSCs [22, 23]. Notably, recent studies have reported that hypoxia-preconditioned MSCs intensify their paracrine abilities [24, 25]. These findings led us to the hypothesis that MSCs cultured under $1 \% \mathrm{O}_{2}$ conditions $\left(1 \% \mathrm{O}_{2}\right.$ MSCs $)$ suppress IRI-induced renal fibrosis and inflammation more strongly than MSCs cultured under normoxic conditions $\left(21 \% \mathrm{O}_{2} \mathrm{MSCs}\right)$.

In this study, using rat- and human-derived bone marrow MSCs, we show that $1 \% \mathrm{O}_{2}$ MSCs ameliorate renal fibrosis and inflammation in vivo and in vitro. We also show that vascular endothelial growth factor (VEGF), hepatocyte growth factor (HGF), and prostaglandin E2 (PGE2) increase in medium of $1 \% \mathrm{O}_{2}$ MSCs compared with that of $21 \% \mathrm{O}_{2}$ MSCs. In addition, we show that inhibition of VEGF suppresses HGF secretion and the anti-fibrotic effect of $1 \% \mathrm{O}_{2}$ MSCs. These findings suggest that the administration of hypoxiapreconditioned MSCs may be a candidate therapy to prevent renal fibrosis and inflammation after AKI.

\section{Methods \\ Animals}

Male Sprague-Dawley (SD) rats (6 and 8 weeks old) were purchased from Charles River Laboratories Japan (Yokohama, Japan). The rats at 6 weeks of age were used to collect bone marrow, and rats at 8 weeks were used to induce IRI. Male CAG-enhanced green fluorescent protein (EGFP)-transgenic SD rats (6 weeks old) were purchased from Japan SLC (Shizuoka, Japan) to collect bone marrow. All experimental procedures were approved by the Institutional Animal Care and Use Committee of Hiroshima University (Hiroshima, Japan) (permit number, A16-83) and conducted in accordance with the Guide for the Care and Use of Laboratory Animals, 8th ed, 2010 (National Institutes of Health, Bethesda, MD, USA).

\section{Preparation of MSCs}

According to previously described methods [26], bone marrow was collected from SD and CAG-EGFPtransgenic $\mathrm{SD}$ rats and cultured in standard culture medium consisting of DMEM (Sigma-Aldrich, St. Louis, MO, USA) with 10\% FBS (Sigma-Aldrich). Cells were passaged four times and used as rat MSCs for transplantation. Human MSCs from bone marrow were purchased from Riken BRC (Ibaraki, Japan). These cells were also cultured in DMEM containing 10\% FBS.

\section{Hypoxic preconditioning}

To perform hypoxic preconditioning, MSCs were cultured in DMEM with 10\% FBS. At 80\% confluence, fresh complete medium was added and hypoxic preconditioning was performed with a Modular Incubator Chamber (MIC 101) (Billups-Rothenberg, San Diego, CA, USA). The cells were incubated in hypoxic conditions $\left(1 \% \mathrm{O}_{2}\right)$ for $24 \mathrm{~h}$.

\section{Experimental animal model}

Renal IRI was performed by transiently clamping the unilateral renal artery. Rats were anesthetized by an intraperitoneal injection of three types of mixed anesthetic agents: medetomidine, midazolam, and butorphanol. After a laparotomy was performed, the left kidney was exposed. Then, the renal pedicle was clamped by atraumatic vascular clamps for $1 \mathrm{~h}$, followed by reperfusion on a heating blanket. After reperfusion, MSCs $\left(5 \times 10^{5}\right.$ cells/rat) in $0.2 \mathrm{ml}$ PBS were injected through the abdominal aorta clamped above and below the left renal artery bifurcation. At 7 or 21 days post-injection, the rats were sacrificed and their kidneys were collected. 


\section{Western blot analysis}

Sample collection and western blotting were performed according to previously described methods [26]. Mouse monoclonal anti- $\alpha$-SMA antibody (Sigma-Aldrich), mouse monoclonal anti-TGF- $\beta 1$ antibody (Santa Cruz Biotechnology, Santa Cruz, CA, USA), mouse monoclonal anti-GAPDH antibody (Sigma-Aldrich), rabbit monoclonal anti-p-Smad2 antibody (Cell Signaling Technology, Danvers, MA, USA), mouse monoclonal anti-Smad2 antibody (Cell Signaling Technology), and mouse monoclonal anti- $\alpha$-Tubulin antibody (Sigma-Aldrich) were used as primary antibodies. Horseradish peroxidase-conjugated goat anti-rabbit immunoglobulin G (Dako, Glostrup, Denmark) or goat anti-mouse immunoglobulin G (Dako) was used as secondary antibodies. SuperSignal West Dura or the Pico system (Thermo Fisher Scientific, Rockford, IL, USA) were used to detect signals. The intensity of each band was measured by ImageJ software (version 1.47v; National Institutes of Health) and normalized to the level of either GAPDH or $\alpha$-tubulin.

\section{Immunohistochemistry analysis}

Immunohistochemical staining was performed according to previously described methods [26]. The following primary antibodies were used: mouse monoclonal anti- $\alpha-$ SMA antibody (Sigma-Aldrich), rabbit polyclonal anticollagen type I antibody (Abcam, Cambridge, UK), rabbit polyclonal anti-collagen type III antibody (Abcam), rabbit polyclonal anti-CD3 antibody (Dako), rabbit polyclonal anti-CD68 antibody (Abcam), rabbit monoclonal anti-CD163 antibody (Abcam), and mouse monoclonal anti-EGFP antibody (Takara Bio, Shiga, Japan). CD3-, CD68-, and CD163-positive cells and positive areas for $\alpha$-SMA and collagen type I and III staining were assessed using Image software by examination of five randomly selected fields $(\times 100)$ of the cortex.

Immunohistochemistry analysis (double immunostaining) Sections of formalin-fixed, paraffin-embedded tissues ( $4 \mu \mathrm{m}$ thick) were de-paraffinized, subjected to heatmediated antigen retrieval in EDTA buffer ( $\mathrm{pH} 9.0)$ at $98^{\circ} \mathrm{C}$ for $40 \mathrm{~min}$, and then blocked in $2.5 \%$ normal horse serum (ImmPRESS Horse Anti-Rabbit IgG Polymer kit; Vector Laboratories, Riverside, CA, USA) at room temperature for $20 \mathrm{~min}$. They were incubated with antiCD163 antibody (Abcam) overnight at $4{ }^{\circ} \mathrm{C}$, followed by incubation with the appropriate secondary antibody (ImmPRESS Horse Anti-Rabbit IgG Polymer kit; Vector Laboratories) at room temperature for $30 \mathrm{~min}$, and then incubated with 3,3'-diaminobenzidine (Sigma-Aldrich) at room temperature for $5 \mathrm{~min}$. After that, they were heated again in EDTA buffer in the same way. They were then blocked, followed by incubation with anti-
CD68 antibody (Abcam) and the secondary antibody in the same way, and next incubated with working solution prepared with Vector SG Peroxidase (HRP) Substrate Kit (Vector Laboratories) at room temperature for $5 \mathrm{~min}$.

\section{Histological analysis}

Sections of formalin-fixed, paraffin-embedded tissues ( $2 \mu \mathrm{m}$ thick) were stained with hematoxylin and eosin (HE), Masson trichrome, and Sirius red to assess histological injury and fibrosis. The areas of interstitial fibrosis were assessed using Lumina Vision (Mitani, Osaka, Japan) by examining five randomly selected fields $(x$ 100) of the cortex.

\section{MSC labeling}

Human MSCs were labeled with CellTracker CM-DiI (Thermo Fisher Scientific) before injection, following the manufacturer's protocols.

\section{Flow cytometric analysis}

Flow cytometric analysis was performed according to previously described methods [26]. The following antibodies were used: anti-human CD29 IgG antibody (BioLegend, San Diego, CA, USA), anti-human CD44 IgG antibody (BioLegend), anti-human CD73 IgG antibody (BioLegend), anti-human CD90 IgG antibody (BioLegend), anti-human CD11b IgG antibody (BioLegend), anti-human CD34 IgG antibody (BioLegend), anti-human CD45 IgG antibody (BioLegend), anti-human HLA-A,B,C IgG antibody (BioLegend), and anti-human HLA-DR IgG antibody (BioLegend). The stained MSCs were analyzed using a BD FACSVerse (Becton, Dickinson and Company, Franklin Lakes, NJ, USA). Data were assessed by FlowJo software (FlowJo, LLC; Ashland, OR, USA).

\section{Preparation of conditioned medium}

To generate conditioned medium from human $1 \% \mathrm{O}_{2}$ MSCs $\left(1 \% \mathrm{O}_{2}\right.$ hMSCs-CM) and human $21 \% \mathrm{O}_{2}$ MSCs $\left(21 \% \mathrm{O}_{2}\right.$ hMSCs-CM), human MSCs $\left(3 \times 10^{5}\right.$ cells/dish $)$ were seeded into $10-\mathrm{cm}$ dishes and grown to $80 \%$ confluence. The culture medium was then substituted with DMEM containing $0.1 \% \mathrm{FBS}$, and the cells were cultured for 24 or $48 \mathrm{~h}$ under hypoxic $\left(1 \% \mathrm{O}_{2}\right)$ or normoxic $(21 \%$ $\mathrm{O}_{2}$ ) conditions. Then, each medium was collected.

\section{Cell culture and treatments}

HK-2 cells were obtained from the American Type Culture Collection (Manassas, VA, USA). The cells were cultured as described previously [26]. After the starvation of HK-2 cells with conditioned medium from human MSCs or DMEM containing 0.1\% FBS for $24 \mathrm{~h}, 10$ $\mathrm{ng} / \mathrm{ml}$ recombinant human TGF- $\beta 1$ (R\&D Systems, Minneapolis, MN, USA) was added to the cells directly. After $30 \mathrm{~min}$ (to investigate protein levels of p-Smad 2) 
or $48 \mathrm{~h}$ (to investigate protein levels of $\alpha$-SMA), HK-2 cells were collected and subjected to in vitro study.

\section{Counting MSCs}

Human MSCs $\left(5 \times 10^{4}\right.$ cells/well $)$ were seeded into sixwell plates and grown to $60-80 \%$ confluence. The culture medium was then substituted with fresh DMEM containing $0.1 \%$ or $10 \% \mathrm{FBS}$, and the cells were cultured under hypoxic $\left(1 \% \mathrm{O}_{2}\right)$ or normoxic $\left(21 \% \mathrm{O}_{2}\right)$ conditions. The number of these cells was counted after 24 or $48 \mathrm{~h}$.

\section{Quantitative real-time reverse-transcription PCR}

RNA extraction and real-time reverse-transcription PCR were performed in accordance with previously described methods [26]. Specific oligonucleotide primers and probes for VEGF (assay ID Hs00900055_m1), HGF (assay ID Hs00300159_m1), and 18S rRNA (endogenous control) were obtained as TaqMan Gene Expression Assays (Applied Biosystems, Foster City, CA, USA). The mRNA levels were standardized by the level of $18 \mathrm{~S}$ rRNA.

\section{ELISAs}

ELISA analyses of VEGF (R\&D Systems), HGF (R\&D Systems), and PGE2 (Enzo Life Science, Farmingdale, NY, USA) were performed following the manufacturers' protocols. Concentrations were normalized to the total protein content.

\section{Transfection of VEGF siRNA}

Human MSCs were transfected with $20 \mathrm{nM}$ siRNA targeting VEGF (sc-461; Applied Biosystems) or negative control siRNA (4390843; Applied Biosystems) using Lipofectamine 2000 Transfection Reagent (Thermo Fisher Scientific). After $24 \mathrm{~h}$, the transfected cells were washed, and fresh complete medium was added. At $80 \%$ confluence, hypoxic preconditioning was performed. Then, the cells were collected and injected into the abdominal aorta. On the other hand, to generate conditioned medium from human $1 \% \mathrm{O}_{2}$ MSCs transfected with VEGF siRNA or negative control siRNA, at $80 \%$ confluence, the transfected cells were cultured in DMEM containing $0.1 \%$ FBS for $48 \mathrm{~h}$ under hypoxic $\left(\begin{array}{ll}1 \% & \mathrm{O}_{2}\end{array}\right)$ conditions. Then, each medium was collected.

\section{Statistical analysis}

Results are expressed as means \pm standard deviations (S.D.). For multiple group comparisons, one-way ANOVA followed by Bonferroni's post hoc test was applied. Comparisons between two groups were analyzed by the Student's $t$ test. $P<0.05$ was considered as statistically significant.

\section{Results}

Hypoxia-preconditioned rat MSCs attenuate IRI-induced renal fibrosis in rats

To investigate the effect of hypoxia-preconditioned rat MSCs on renal fibrosis, we first examined the expression of $\alpha$-SMA and TGF- $\beta 1$ in the IRI model that had been injected with PBS, $5 \times 10^{5}$ rat MSCs cultured under normoxic conditions $\left(21 \% \mathrm{O}_{2}\right.$ rMSCs $)$, or under $1 \% \mathrm{O}_{2}$ conditions $\left(1 \% \mathrm{O}_{2}\right.$ rMSCs $)$ at 21 days post-IRI. As shown in Fig. $1 \mathrm{a}$ and $\mathrm{b}$, protein levels of $\alpha$-SMA and TGF- $\beta 1$ were remarkably increased in IRI rats injected with PBS (PBS group). Such expression was suppressed in IRI rats injected with $21 \% \mathrm{O}_{2}$ rMSCs $\left(21 \% \mathrm{O}_{2}\right.$ rMSC group), and further suppression was observed in those injected with $1 \% \mathrm{O}_{2}$ rMSCs $\left(1 \% \mathrm{O}_{2}\right.$ rMSC group). Similarly, immunostaining revealed that the $\alpha$-SMA-positive area was reduced in the $1 \% \mathrm{O}_{2}$ rMSC group compared with the $21 \% \mathrm{O}_{2}$ rMSC group (Fig. 1c, d). Furthermore, immunostaining revealed that collagen type I- and III-positive areas were more significantly suppressed in the $1 \% \mathrm{O}_{2}$ rMSC group than in the $21 \% \mathrm{O}_{2}$ rMSC group (Fig. 1c, d). Moreover, we performed $\mathrm{HE}$ staining at 7 and 21 days post-IRI. HE staining showed tubular dilatation, tubular cast formation, and diffused infiltration of inflammatory cells at 7 days post-IRI. These tubulointerstitial injuries were suppressed by the administration of $21 \% \mathrm{O}_{2}$ rMSCs or $1 \% \mathrm{O}_{2}$ rMSCs, with no significant difference between them (Additional file 1). Further progression of tubulointerstitial injuries and tubular atrophy were found at 21 days post-IRI. The progression of tubulointerstitial injuries and tubular atrophy were suppressed by the administration of $21 \% \mathrm{O}_{2}$ rMSCs, and further suppression was observed in the $1 \% \mathrm{O}_{2}$ rMSC group (Fig. 1e). Masson trichrome staining and Sirius red staining showed only slight interstitial fibrosis at 7 days post-IRI (Additional file 1), so we considered that the rat model at 7 days post-IRI was not suitable for evaluating the anti-fibrotic effect of MSCs. At 21 days post-IRI, further progression of interstitial fibrosis was observed in the PBS group and was attenuated by MSC treatment, particularly in the $1 \% \mathrm{O}_{2}$ rMSC group (Fig. 1e, f).

Hypoxia-preconditioned rat MSCs suppress the infiltration of inflammatory cells in IRI rats

To assess the anti-inflammatory effect of hypoxiapreconditioned rat MSCs, we examined the expression of CD3 (T cell marker), CD68 (M1 and M2 macrophage marker), and CD163 (M2 macrophage marker) at 7 days post-IRI. Immunohistochemical staining revealed that the accumulation of CD3- and CD68-positive cells was increased in the PBS group (Fig. 2a, b). The infiltration of these cells was suppressed in the $21 \% \mathrm{O}_{2}$ rMSC group, whereas it was suppressed more significantly in the $1 \% \mathrm{O}_{2}$ rMSC group (Fig. 2a, b). In contrast, the 
a
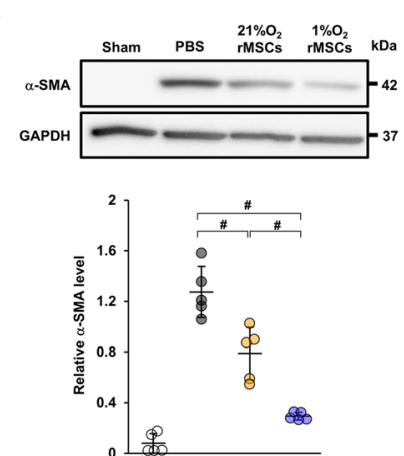

OSham OPBS $\mathrm{O}_{21} 1 \mathrm{O}_{2}$ rMSCs $01 \% \mathrm{O}_{2}$ rMSCs b
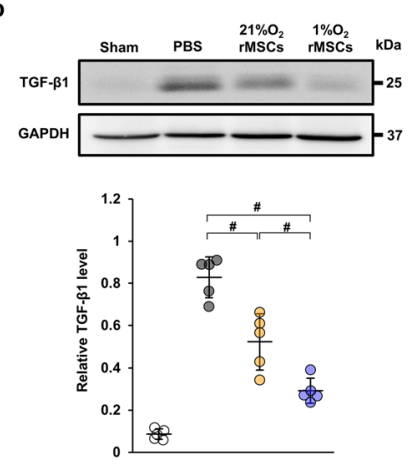

OSham OPBS $\mathrm{O}_{21} 1 \mathrm{O}_{2}$ rMSCs $\mathrm{O}_{1} \% \mathrm{O}_{2}$ rMscs

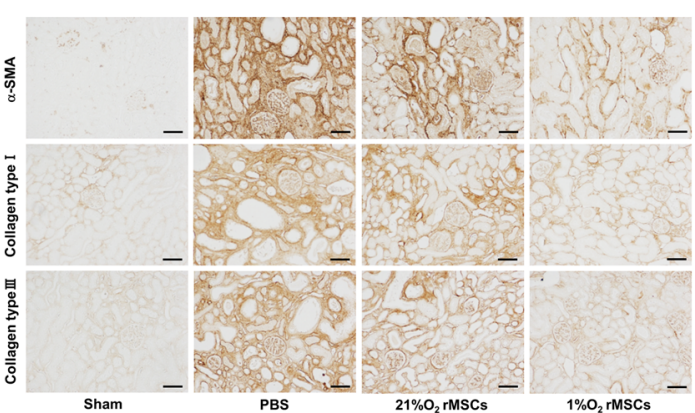

d
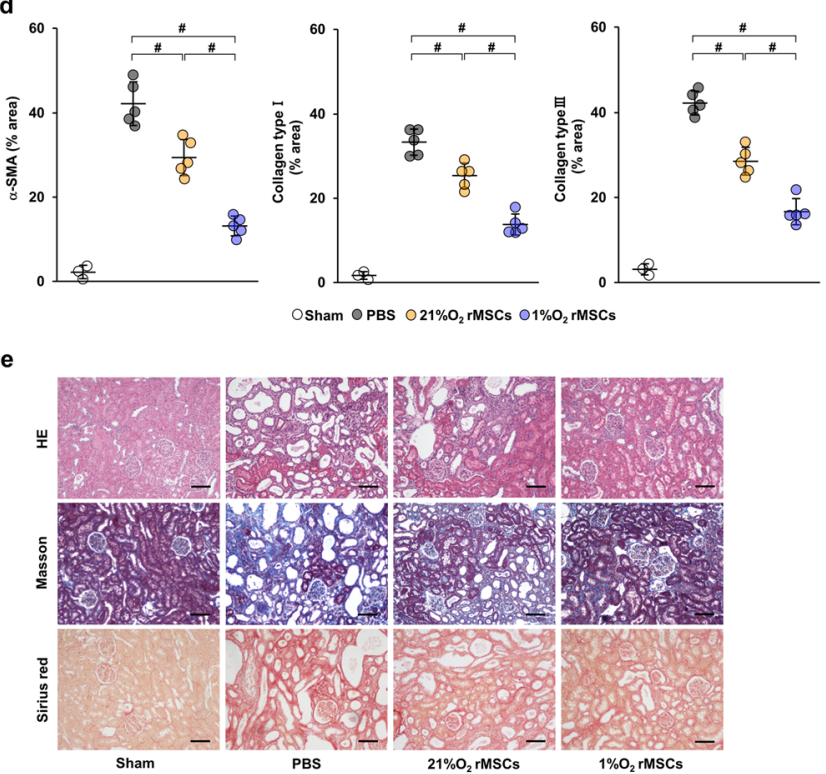

f

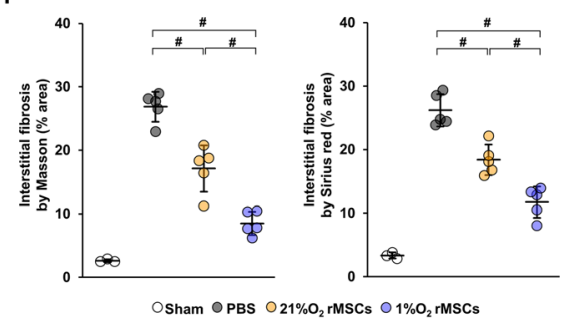

Fig. 1 (See legend on next page.) 
(See figure on previous page.)

Fig. $11 \% \mathrm{O}_{2}$ rMSCs suppress renal fibrosis more significantly than $21 \% \mathrm{O}_{2}$ rMSCs in IRI rats. a, $\mathbf{b}$ Western blot analysis of a-SMA and TGF- $\beta 1$ in the kidney cortex of IRI rats at day 21 post-IRI. Graphs show densitometric analyses of a-SMA and TGF- $\beta 1$ expression levels normalized to the GAPDH expression level. $\mathbf{c}$ Representative immunohistochemical staining of a-SMA and collagen type I and III in kidney sections at day 21 post-IRI (scale bar $=100 \mu \mathrm{m}$ ). $\mathbf{d}$ Quantification of a-SMA- and collagen type I- and III-positive areas as percentages of the total area. e Representative images of HE, Masson trichrome, and Sirius red staining in kidney sections at day 21 post-IRI (scale bar $=100 \mu \mathrm{m}$ ). $\mathbf{f}$ Quantification of interstitial fibrosis area as percentages of the total area. Data are means \pm S.D.

$\# P<0.01$ (one-way ANOVA followed by Bonferroni's post hoc test)

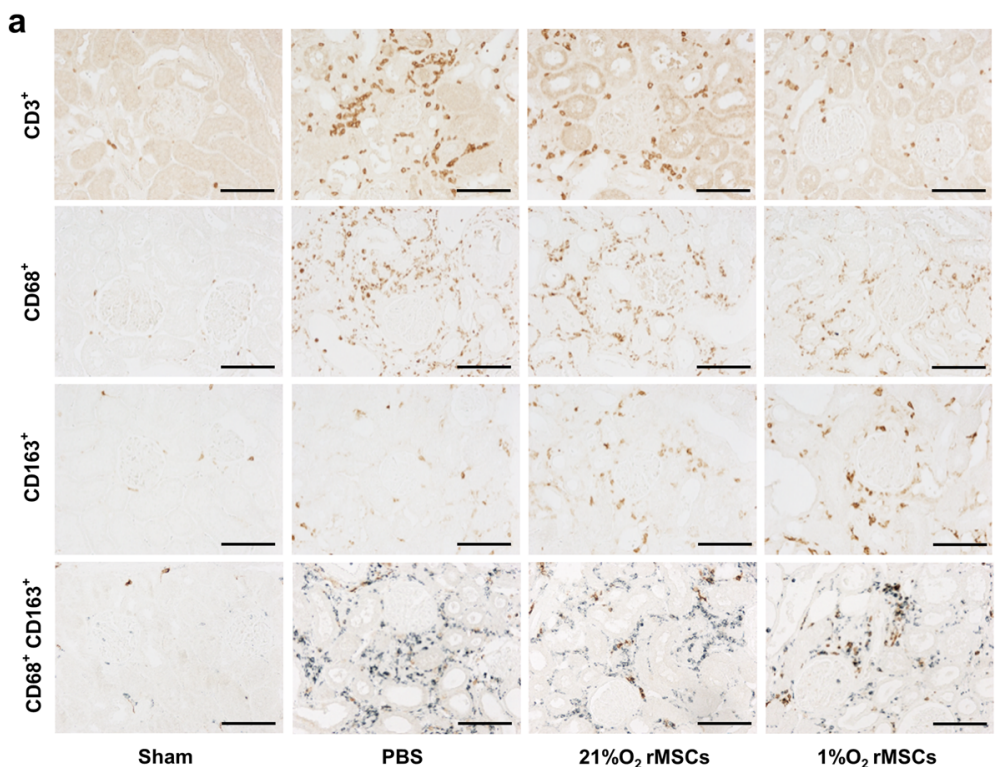

b
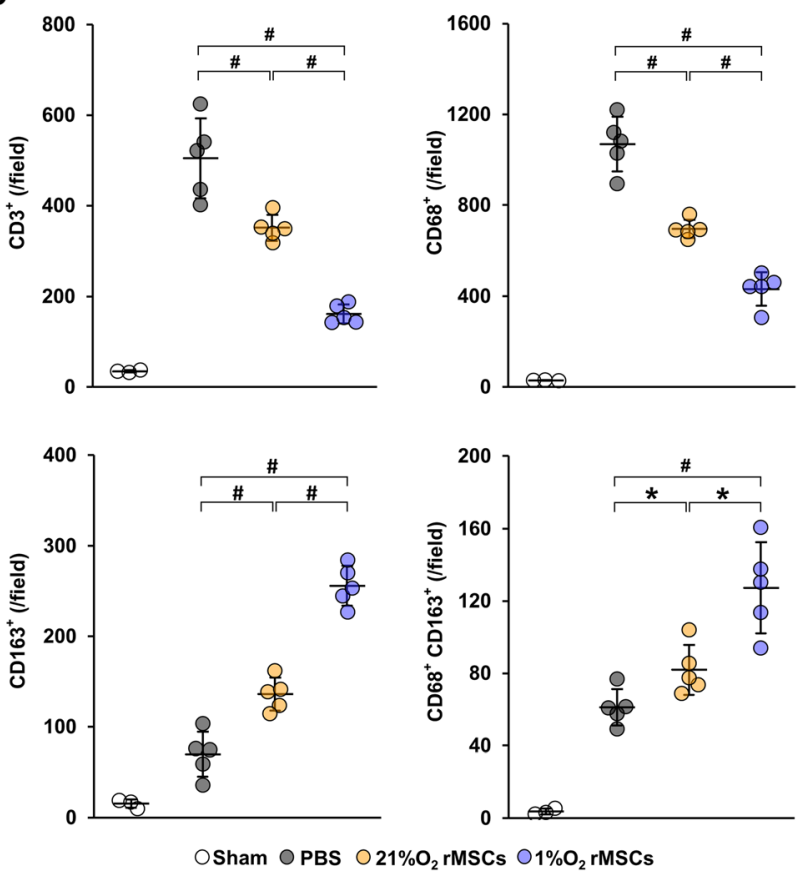

Fig. $21 \% \mathrm{O}_{2}$ rMSCs attenuate renal inflammation more strongly than $21 \% \mathrm{O}_{2} \mathrm{rMSCS}$ in IRI rats. a Representative images of immunostaining of CD3, CD68, and CD163 and double immunostaining of CD163 (brown) and CD68 (blue) in the kidney sections at day 7 post-IRI (scale bar = $100 \mu \mathrm{m})$. b Quantification of CD3-, CD68-, and CD163-positive cells and double-positive (CD68 and CD163) cells. Data are means \pm S.D. ${ }^{\#} P<0.01$, *P 0.05 (one-way ANOVA followed by Bonferroni's post hoc test) 
expression of CD163, an immunosuppressive macrophage marker, was increased in the $21 \% \mathrm{O}_{2}$ rMSC group, and a further increase was observed in the $1 \% \mathrm{O}_{2}$ rMSC group (Fig. 2a, b). In addition, we performed double immunostaining for CD68 and CD163 to identify M2 macrophages. Positive cells for both CD68 and CD163 were increased in the $21 \% \mathrm{O}_{2}$ rMSC group, and a further increase was observed in the $1 \% \mathrm{O}_{2}$ rMSC group (Fig. 2a, b).

\section{Hypoxia-preconditioned human MSCs also attenuate renal fibrosis in IRI rats}

To investigate whether hypoxia-preconditioned human MSCs also exerted therapeutic effects on renal fibrosis, we next injected PBS, $5 \times 10^{5}$ human MSCs cultured under normoxic conditions $\left(21 \% \mathrm{O}_{2}\right.$ hMSCs $)$, or under $1 \% \mathrm{O}_{2}$ conditions $\left(1 \% \mathrm{O}_{2}\right.$ hMSCs $)$ into the IRI model $\left(21 \% \mathrm{O}_{2}\right.$ hMSC and $1 \% \mathrm{O}_{2}$ hMSC groups, respectively). Although the upregulation of $\alpha$-SMA and TGF- $\beta 1$ in the PBS group was suppressed in the $21 \% \mathrm{O}_{2}$ hMSC group, their expression in the $1 \% \mathrm{O}_{2} \mathrm{hMSC}$ group was reduced more significantly than in the $21 \% \mathrm{O}_{2}$ hMSC group at 21 days post-IRI (Fig. 3a, b). Similarly, immunostaining revealed that the $\alpha$-SMA-positive area was reduced in the $1 \% \mathrm{O}_{2}$ hMSC group compared with the $21 \% \mathrm{O}_{2}$ hMSC group (Fig. 3c, d). Furthermore, immunostaining revealed that collagen type I- and III-positive areas were more significantly suppressed in the $1 \% \mathrm{O}_{2}$ hMSC group than in the $21 \% \mathrm{O}_{2}$ hMSC group (Fig. 3c, d). Moreover, $\mathrm{HE}$ staining showed that tubulointerstitial injuries were more significantly suppressed in the $1 \% \mathrm{O}_{2}$ hMSC group than in the $21 \% \mathrm{O}_{2}$ hMSC group (Fig. 3e). Masson trichrome staining and Sirius red staining revealed that the area of interstitial fibrosis was reduced by MSC treatment, particularly in the $1 \% \mathrm{O}_{2}$ hMSC group (Fig. 3e, f).

\section{Hypoxic preconditioning does not change the immunophenotype of MSCs}

It has been reported that the preconditioning of MSCs with hypoxia enhanced their migration capacity [25, 27]. Therefore, we studied whether hypoxic preconditioning enhances the engraftment capacity of MSCs using MSCs from male CAG-EGFP-transgenic SD rats. EGFPpositive cells were assessed as the average of ten randomly selected fields $(\times 100)$ for each rat at 21 days postIRI $\left(1 \% \mathrm{O}_{2}\right.$ rMSC group and $21 \% \mathrm{O}_{2}$ rMSC group, $\left.n=5\right)$. Immunohistochemical staining for EGFP revealed that the number of EGFP-positive cells was $10 \pm 1$ cells/10 fields in the $1 \% \mathrm{O}_{2}$ rMSC group and $10 \pm 1$ cells $/ 10$ fields $(\times 100)$ in the $21 \% \mathrm{O}_{2}$ rMSC group (Additional file $2 \mathrm{a}$ ). These results suggest that hypoxic preconditioning does not increase the engraftment capacity of MSCs. In contrast, we found that anti-fibrotic ability was almost equal in $1 \% \mathrm{O}_{2}$ rMSCs and $1 \% \mathrm{O}_{2}$ hMSCs. Therefore, we investigated their engraftment using rMSCs from EGFP- transgenic rats and DiI-labeled hMSCs. EGFP-expressing rMSCs and DiI-labeled hMSCs were both observed in the kidney at day 21 post-IRI (Additional file 2a, b). Next, to assess whether hypoxic preconditioning affected their immunophenotype, we analyzed expression levels of cell surface markers using flow cytometry. Although human leukocyte antigen (HLA) expression plays an important role in the allogeneic immune response, the expression level of HLA-A,B,C did not differ between $21 \% \mathrm{O}_{2}$ hMSCs and $1 \% \mathrm{O}_{2}$ hMSCs (Additional file 3). Moreover, HLA-DR expression was not observed in both $21 \% \mathrm{O}_{2}$ hMSCs and $1 \% \mathrm{O}_{2}$ hMSCs (Additional file 3). Similarly, $21 \% \mathrm{O}_{2}$ hMSCs and $1 \% \mathrm{O}_{2}$ hMSCs expressed comparable levels of standard MSC markers, such as CD29, CD44, CD73, and CD90 (Additional file 3), and did not express MSC negative markers such as CD11b, CD34, and CD45 (Additional file 3).

\section{Conditioned medium from hypoxia-preconditioned human MSCs suppresses fibrotic changes through inhibition of TGF- $\beta / S m a d$ signaling}

To identify the direct effect of hypoxia-preconditioned MSCs on TGF- $\beta /$ Smad signaling, we examined the expression of phosphorylated Smad2 and $\alpha$-SMA in TGF$\beta 1$-stimulated HK- 2 cells. We prepared $21 \% \mathrm{O}_{2}$ hMSC$\mathrm{CM}$ and $1 \% \mathrm{O}_{2} \mathrm{hMSC}-\mathrm{CM}$, and then stimulated HK-2 cells with TGF- $\beta 1$ with and without each conditioned medium. The protein level of phosphorylated Smad2 was increased by TGF- $\beta 1$ stimulation. The upregulation of phosphorylated Smad2 was more significantly suppressed by $1 \% \mathrm{O}_{2}$ hMSC-CM than both normal medium and $21 \% \mathrm{O}_{2}$ hMSC-CM (Fig. 4a). Similar results were observed for $\alpha$-SMA protein expression (Fig. $4 b$ ).

\section{Hypoxia-preconditioned human MSCs enhance the production of VEGF, HGF, and PGE2}

MSCs reportedly secrete several humoral factors that promote tissue repair in a paracrine manner [22, 23]. To investigate whether hypoxia-preconditioned MSCs enhanced the secretion of these factors, we measured the concentrations of VEGF, HGF, and PGE2 in conditioned medium using ELISA kits. First, we confirmed that the numbers of both $1 \% \mathrm{O}_{2}$ hMSCs and $21 \% \mathrm{O}_{2}$ hMSCs were hardly increased because MSC-CM was obtained using 0.1\% FBS-containing medium (Fig. 5a). As shown in Fig. $5 \mathrm{~b}$, we found upregulation of VEGF, HGF, and PGE2 in $1 \% \mathrm{O}_{2}$ hMSC-CM compared with $21 \% \mathrm{O}_{2}$ hMSC-CM. Next, we measured the expression levels of VEGF and HGF mRNA of hMSCs cultured with 10\% FBScontaining medium. The number of $1 \% \mathrm{O}_{2}$ hMSCs cultured with $10 \%$ FBS increased, as did that of $21 \%$ $\mathrm{O}_{2}$ hMSCs cultured with 10\% FBS (Additional file 4a). In these conditions, we found upregulation of VEGF mRNA levels in the $1 \% \mathrm{O}_{2}$ hMSCs compared with 
a
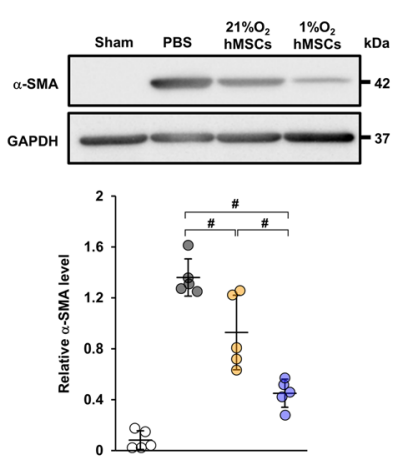

OSham O PBS $\bigcirc 21 \% \mathrm{O}_{2} \mathrm{hMSCs} 01 \% \mathrm{O}_{2} \mathrm{hMSCs}$

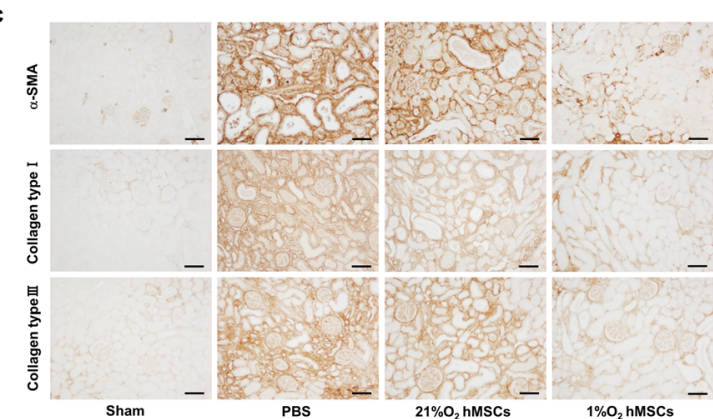

d

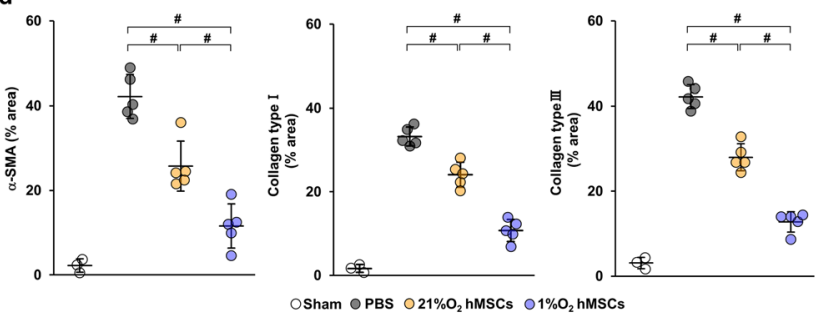

e

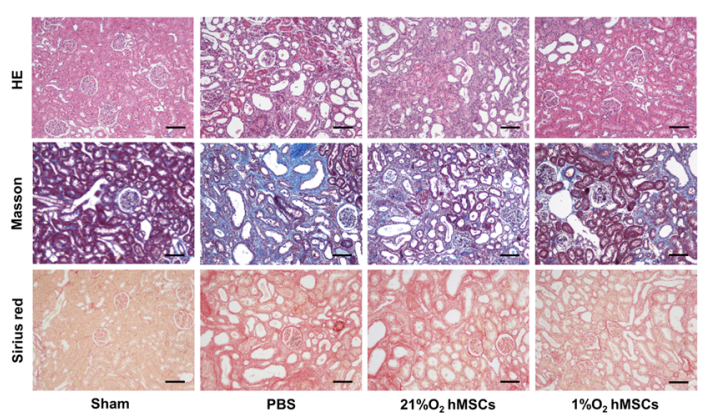

f

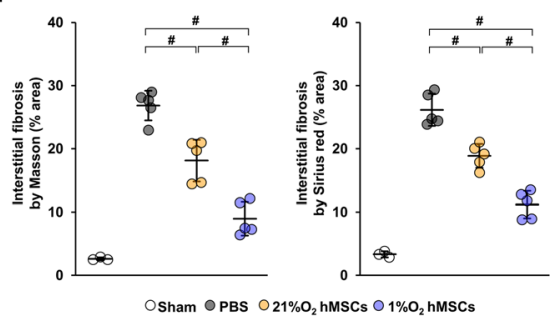

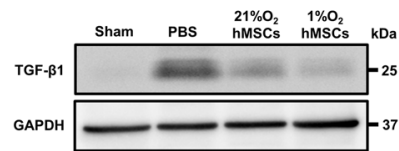

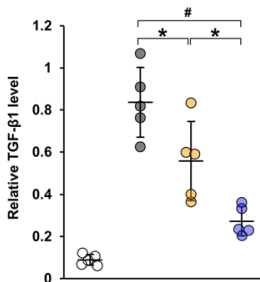

$21 \% \mathrm{O}_{2} \mathrm{mac} \mathrm{O}_{1 \%}$

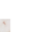


(See figure on previous page.)

Fig. $31 \% \mathrm{O}_{2}$ hMSCs suppress renal fibrosis more significantly than $21 \% \mathrm{O}_{2}$ hMSCs in IRI rats. a, $\mathbf{b}$ Western blot analysis of a-SMA and TGF- $\beta 1$ in the kidney cortex of IRI rats at day 21 post-IRI. Graph shows densitometric analysis of a-SMA and TGF- $\beta 1$ expression levels normalized to the GAPDH expression level. c Representative immunohistochemical staining of a-SMA and collagen type I and III in kidney sections at day 21 postIRI (scale bar $=100 \mu \mathrm{m}$ ). d Quantification of a-SMA- and collagen type I- and III-positive areas as percentages of the total area. e Representative images of HE, Masson trichrome, and Sirius red staining in kidney sections at day 21 post-IRI (scale bar $=100 \mu m$ ). $\mathbf{f}$ Quantification of interstitial fibrosis area as percentages of the total area. Data are means \pm S.D. ${ }^{\#} P<0.01,{ }^{*} P<0.05$ (one-way ANOVA followed by Bonferroni's post hoc test)

those in the $21 \% \mathrm{O}_{2}$ hMSCs (Additional file $4 \mathrm{~b}$ ). However, there were no significant differences in the VEGF mRNA levels between hMSCs cultured under $1 \% \mathrm{O}_{2}$ conditions for $24 \mathrm{~h}\left(1 \% \mathrm{O}_{2} 24 \mathrm{~h}\right.$ hMSCs $)$ and $48 \mathrm{~h}\left(1 \% \mathrm{O}_{2}\right.$ $48 \mathrm{~h}$ hMSCs). Conversely, after both 24 and $48 \mathrm{~h}$, there were no significant differences in HGF mRNA levels between $1 \% \mathrm{O}_{2}$ hMSCs and $21 \% \mathrm{O}_{2}$ hMSCs (Additional file 4c).

Knockdown of VEGF in hypoxia-preconditioned human MSCs reduces the secretion of HGF and the anti-fibrotic effect in TGF- $\beta 1$-stimulated HK-2 cells

The expression level of VEGF in $1 \% \mathrm{O}_{2}$ hMSCs-CM was higher than in $21 \% \mathrm{O}_{2}$ hMSCs-CM. Therefore, to assess whether VEGF in $1 \% \mathrm{O}_{2}$ hMSCs-CM had an anti-fibrotic effect, we investigated $1 \% \mathrm{O}_{2}$ hMSCs transfected with VEGF siRNA (VEGF siRNA/1\% $\mathrm{O}_{2}$ hMSCs) or negative control siRNA (NC siRNA/1\% $\mathrm{O}_{2}$ hMSCs). We confirmed the successful knockdown of VEGF in siRNA-transfected $1 \% \mathrm{O}_{2}$ hMSCs by analyzing their conditioned medium (Fig. 6a). We next investigated the expression of HGF in the condition medium. As shown in Fig. 6b, ELISA analysis revealed that the expression of HGF was lower in conditioned medium from VEGF siRNA $/ 1 \% \mathrm{O}_{2}$ hMSCs (VEGF siRNA $/ 1 \% \mathrm{O}_{2}$ hMSC-CM) compared with that from $\mathrm{NC}$ siRNA $/ 1 \% \mathrm{O}_{2}$ hMSCs (NC siRNA $/ 1 \% \mathrm{O}_{2}$ hMSC-CM). Therefore, we examined whether VEGF was the upstream effector of HGF in hMSCs. As shown in Fig. 6c, we found that VEGF increased HGF expression after $72 \mathrm{~h}$ in a dose-dependent manner. Moreover, to elucidate the effect of VEGF on TGF- $\beta 1$ signaling, we investigated the effect of $\mathrm{NC}$ siRNA $/ 1 \% \mathrm{O}_{2}$ hMSC-CM and VEGF siRNA $/ 1 \% \mathrm{O}_{2}$ hMSC-CM on $\alpha$-SMA expression in TGF- $\beta 1$-stimulated HK2 cells. NC siRNA $/ 1 \% \mathrm{O}_{2}$ hMSC-CM suppressed TGF- $\beta 1$-induced the expression of $\alpha$-SMA, whereas a
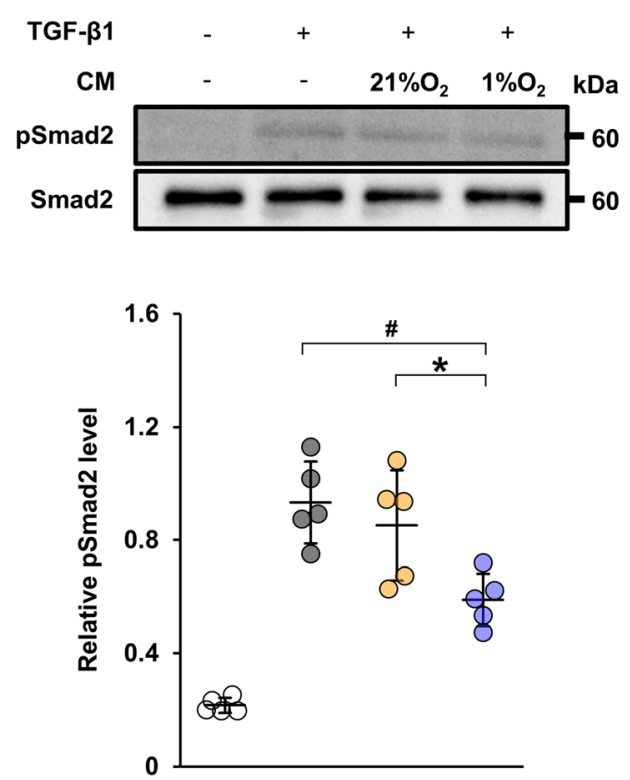

OControl O DMEM $\bigcirc 21 \% \mathrm{O}_{2} \mathrm{hMSC}-\mathrm{CM} \bigcirc 1 \% \mathrm{O}_{2} \mathrm{hMSC}-\mathrm{CM}$ b
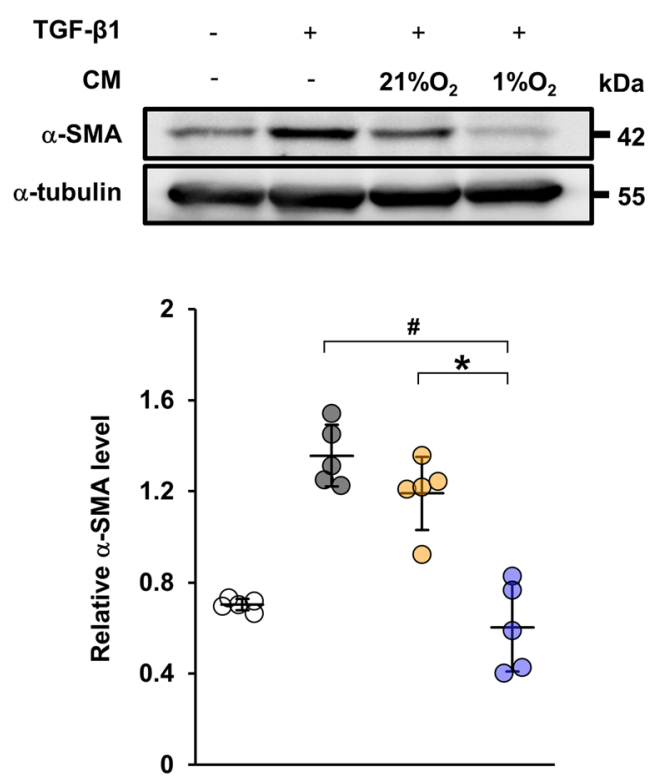

OControl O DMEM $\bigcirc 21 \% \mathrm{O}_{2} \mathrm{hMSC}-\mathrm{CM} \bigcirc 1 \% \mathrm{O}_{2} \mathrm{hMSC}-\mathrm{CM}$

Fig. 4 Conditioned medium from $1 \% \mathrm{O}_{2}$ hMSCs inhibit TGF- $\beta 1$-induced fibrotic changes in HK-2 cells strongly. a Western blot analysis of phosphorylated Smad2 (pSmad2) in HK-2 cells stimulated with TGF- $\beta 1$ for 30 min. Graph shows densitometric analysis of the pSmad2 expression level normalized to the Smad2 expression level. $\mathbf{b}$ Western blot analysis of a-SMA in HK-2 cells stimulated with TGF- $\beta 1$ for 48 h. Graph shows densitometric analysis of the a-SMA expression level normalized to the a-tubulin expression level. Data are means \pm S.D. ${ }^{*} P<0.01,{ }^{*} P<0.05$ (one-way ANOVA followed by Bonferroni's post hoc test) 


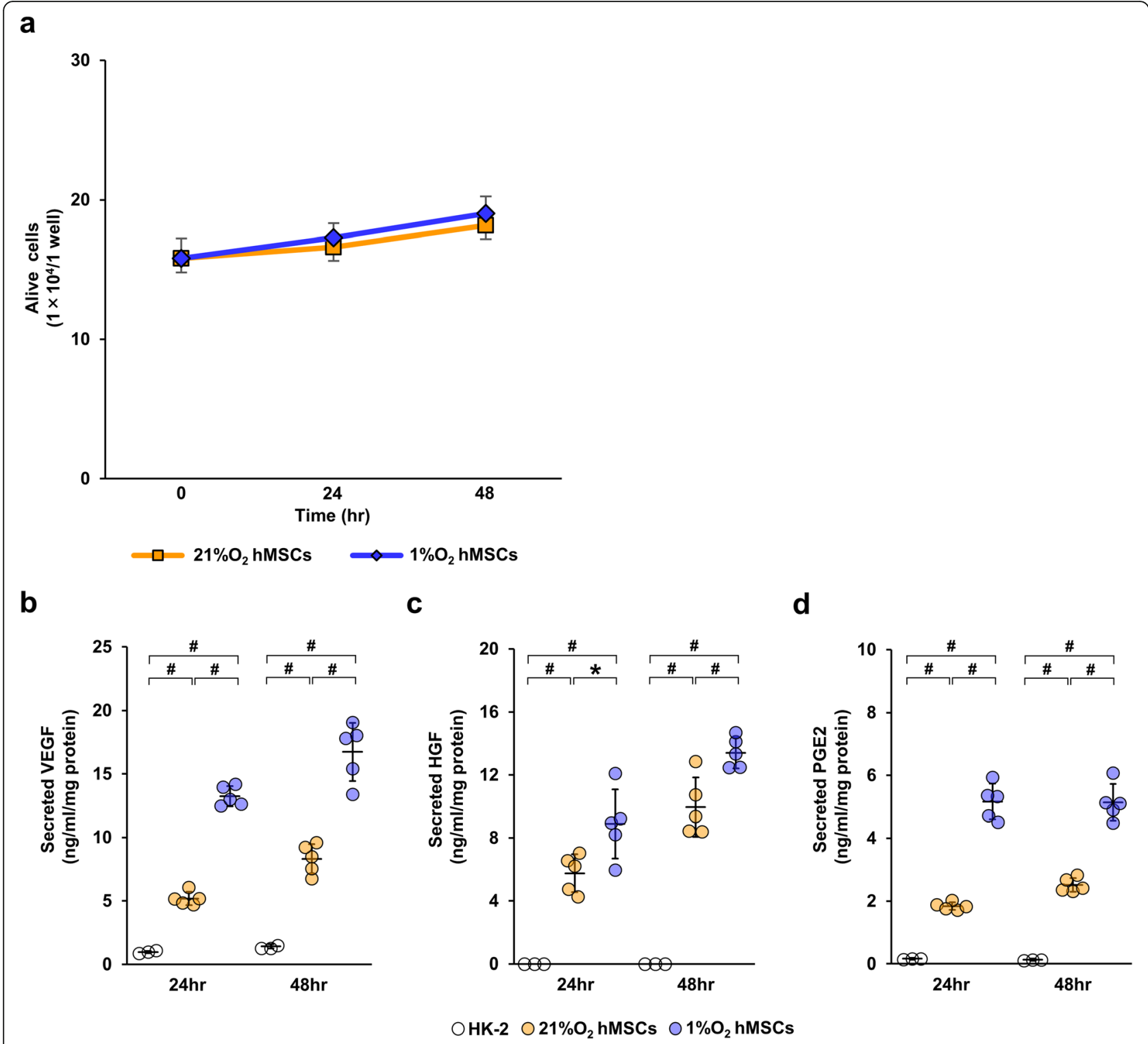

Fig. $51 \% \mathrm{O}_{2}$ hMSCs increase secretion of VEGF, HGF, and PGE2. a Graph showing the number of alive MSCs under normoxic conditions or $1 \% \mathrm{O}_{2}$ conditions. Concentrations of vascular endothelial growth factor (VEGF) (b), hepatocyte growth factor (HGF) (c), and prostaglandin E2 (PGE2) (d) in each CM were measured using ELISA kits. Data are means \pm S.D. ${ }^{\#} P<0.01,{ }^{*} P<0.05$ (one-way ANOVA followed by Bonferroni's post hoc test)

VEGF siRNA $/ 1 \% \mathrm{O}_{2}$ hMSC-CM attenuated this inhibitory effect (Fig. 6d).

\section{Knockdown of VEGF in hypoxia-preconditioned human MSCs reduces the anti-fibrotic effect in IRI rats}

To assess the effect of VEGF from $1 \% \mathrm{O}_{2}$ hMSCs on renal fibrosis, we injected $1 \% \mathrm{O}_{2}$ hMSCs transfected with VEGF siRNA or negative control siRNA into IRI rats (VEGF siRNA $/ 1 \% \mathrm{O}_{2}$ hMSC and $\mathrm{NC}$ siRNA $/ 1 \% \mathrm{O}_{2}$ hMSC groups, respectively). As shown in Fig. 7a and b, the protein levels of $\alpha$-SMA and TGF- $\beta 1$ were markedly increased in the PBS group and their upregulation was significantly inhibited in the NC siRNA/ $1 \% \mathrm{O}_{2}$ hMSC group. However, their beneficial effect was weakened in the VEGF siRNA/1\% $\mathrm{O}_{2}$ hMSC group. Immunostaining also revealed that the $\alpha$ SMA-positive area was significantly reduced in the NC siRNA $/ 1 \% \mathrm{O}_{2}$ hMSC group, whereas it was decreased in the VEGF siRNA $/ 1 \% \mathrm{O}_{2}$ hMSC group (Fig. 7c, d). Similarly, collagen type I- and III-positive areas were markedly suppressed in the NC siRNA/ $1 \% \mathrm{O}_{2}$ hMSC group, whereas the anti-fibrotic effect was reduced in the VEGF siRNA/ $1 \% \mathrm{O}_{2}$ hMSC group (Fig. 7c, d).

\section{Discussion}

This study has revealed that the administration of $1 \% \mathrm{O}_{2}$ MSCs ameliorates renal fibrosis and inflammation in IRI 


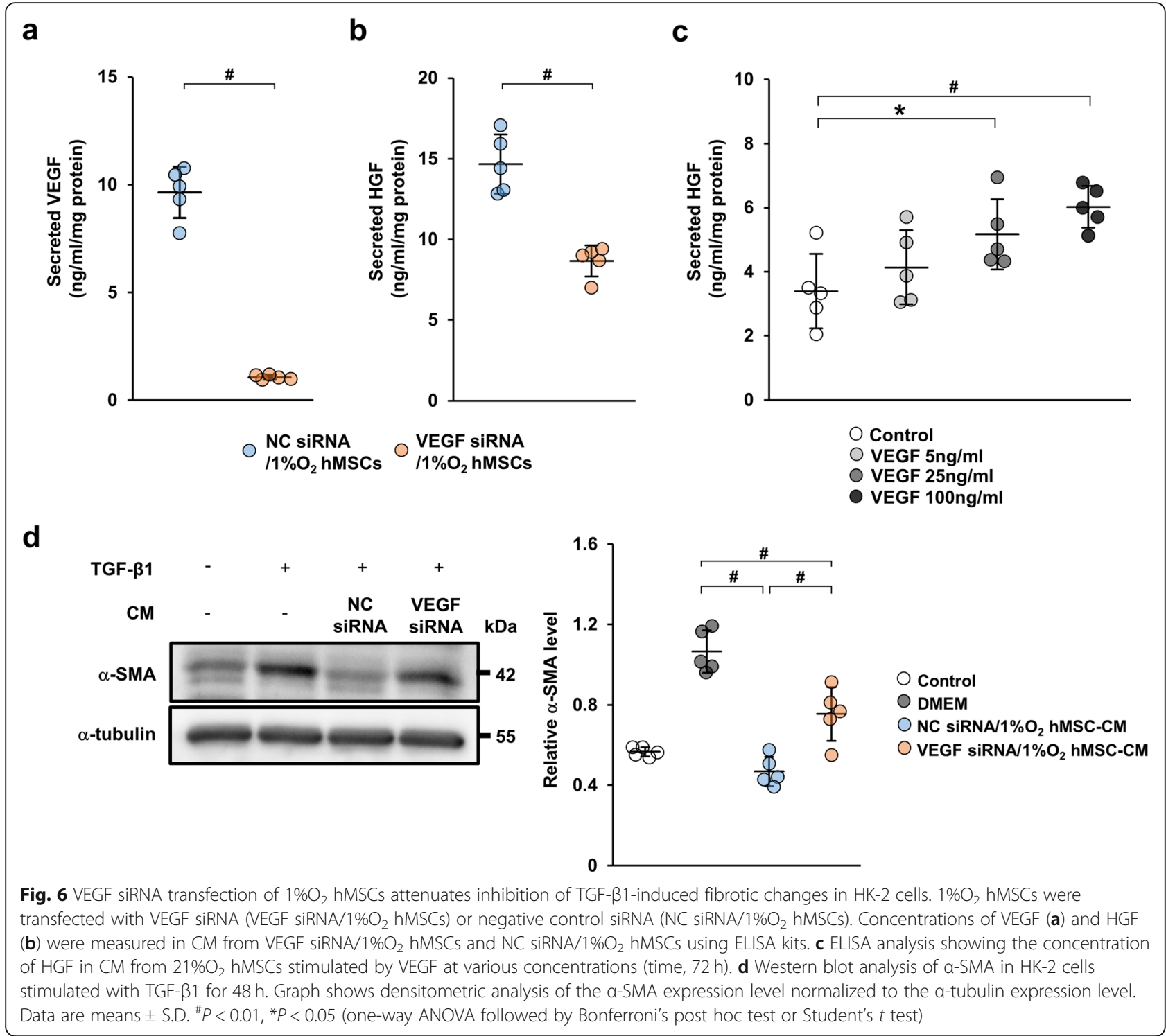

rats. These beneficial effects are almost equivalent for rat- and human-derived bone marrow MSCs. In comparison with $21 \% \mathrm{O}_{2} \mathrm{MSCs}, 1 \% \mathrm{O}_{2}$ MSCs further inhibited TGF- $\beta /$ Smad signaling. Furthermore, $1 \% \mathrm{O}_{2}$ MSCs enhanced VEGF secretion, whereas knockdown of VEGF reduced the anti-fibrotic effect of $1 \% \mathrm{O}_{2}$ MSCs. These findings suggest that hypoxia-preconditioned MSCs enhance renoprotective effects by intensifying their paracrine abilities.

Previous studies have demonstrated that hypoxiapreconditioned MSCs attenuate tissue damage, which is mostly evaluated at the acute phase after tissue injury, but not at the chronic phase [28-30]. Pathologically, IRI induces acute tubular necrosis that follows the production of cytokines, including TGF- $\beta 1$, which plays a major role in the progression of chronic renal damage [14-16]. These findings suggest that the therapeutic effects of
$1 \% \mathrm{O}_{2}$ MSCs are the result of ameliorating AKI. However, our data also showed that $1 \% \mathrm{O}_{2}$ MSCs enhanced the ability to produce anti-fibrotic and anti-inflammatory humoral factors and that medium from $1 \% \mathrm{O}_{2}$ MSCs inhibited TGF- $\beta 1$-induced fibrotic changes. Moreover, we found that MSCs existed in the kidney at 21 days after IRI. Taken together, hypoxia-preconditioned MSCs confer the ability to suppress chronic tissue damage after AKI.

As a mechanism by which MSCs exert an anti-fibrotic effect, we previously found that conditioned medium from MSCs inhibits TGF- $\beta /$ Smad signaling [31]. The presenting data showed that conditioned medium from $1 \% \mathrm{O}_{2}$ MSCs further suppressed TGF- $\beta 1$-induced phosphorylation of Smad2 and $\alpha$-SMA in HK-2 cells compared with $21 \% \mathrm{O}_{2}$ MSCs. In terms of inflammation, we found that $1 \% \mathrm{O}_{2}$ MSCs enhanced immunosuppressive abilities in IRI rats. Among humoral factors, PGE2 is 
a
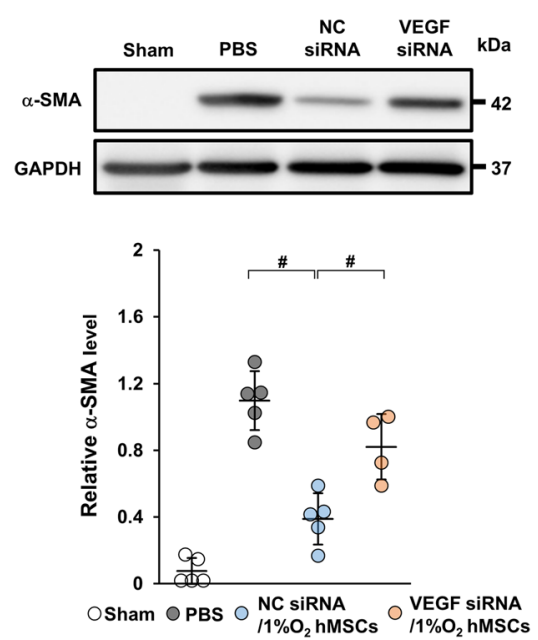

b
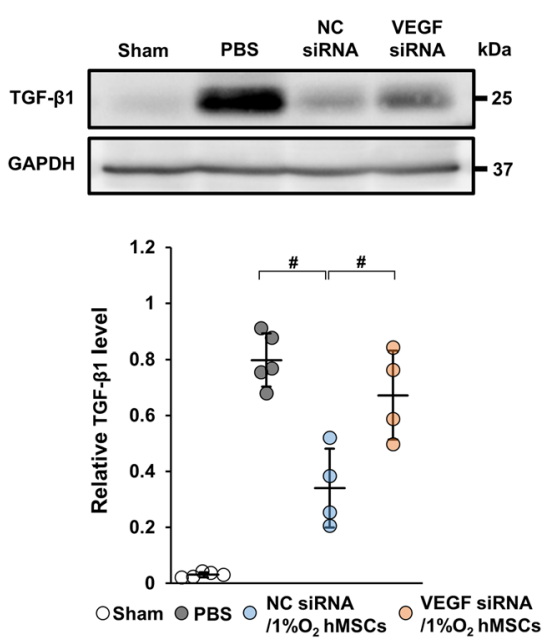

C

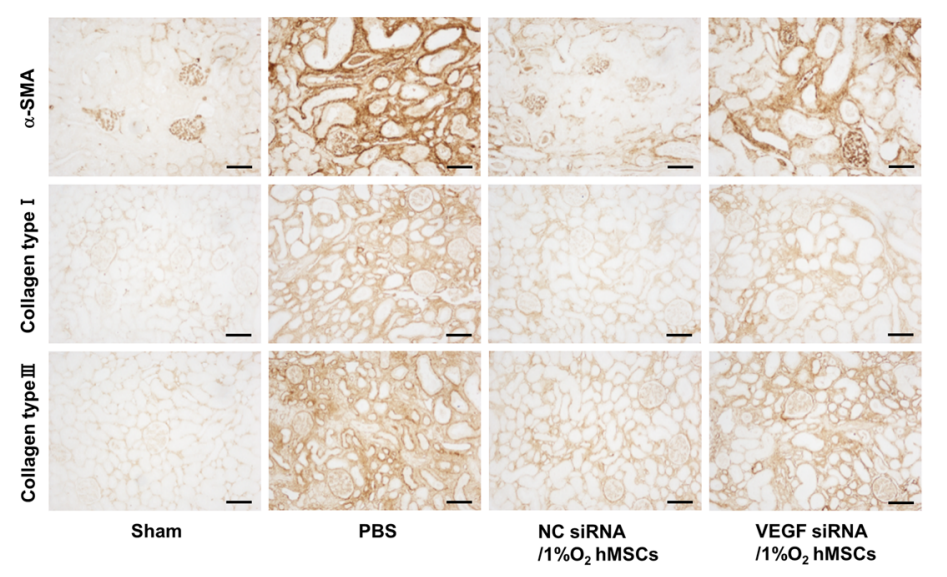

d

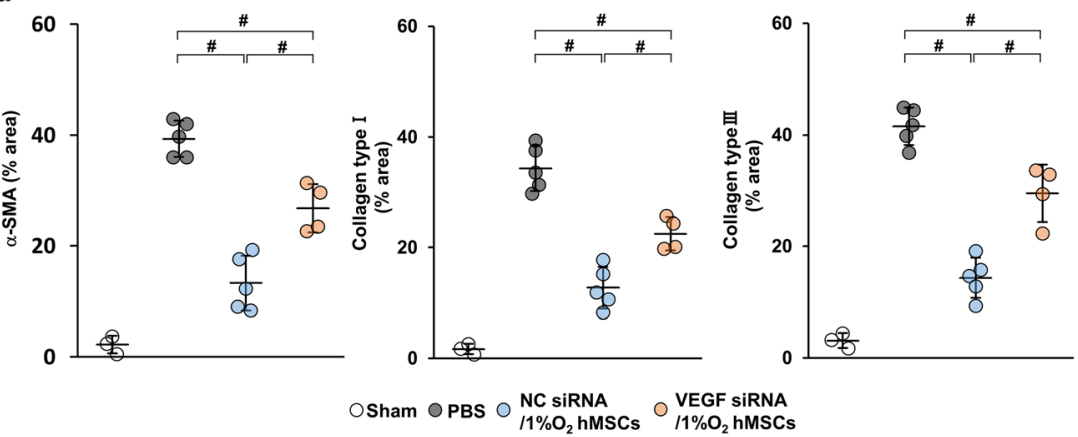

Fig. 7 VEGF siRNA transfection attenuates the anti-fibrotic effect of $1 \% \mathrm{O}_{2}$ hMSCs in IRI rats. $\mathbf{a}$, $\mathbf{b}$ Western blot analysis of a-SMA and TGF- $\beta 1$ in the kidney cortex of IRI rats at day 21 post-IRI. Graphs show densitometric analysis of a-SMA and TGF- $\beta 1$ expression levels normalized to the GAPDH expression level. c Representative immunohistochemical staining of a-SMA and collagen type I and III in kidney sections at day 21 post-IRI (scale bar $=100 \mu \mathrm{m})$. d Quantification of a-SMA and collagen type I- and III-positive areas as percentages of the total area. Data are means \pm S.D. ${ }^{\#} P<0.01$ (one-way ANOVA followed by Bonferroni's post hoc test)

reportedly secreted from MSCs, playing an important role in changing the phenotype of macrophages from proinflammatory M1 to immunosuppressive M2 [32]. Indeed, we demonstrated that $1 \% \mathrm{O}_{2}$ MSCs enhanced PGE2 secretion and that the administration of $1 \% \mathrm{O}_{2}$ MSCs not only suppressed the infiltration of inflammatory cells, but also increased M2 macrophages in IRI rats. Considering that chronic inflammation is well recognized to participate in the progression of renal fibrosis $[11,14-16]$, these results suggest that, in addition to the inhibition of TGF- $\beta /$ Smad signaling, the enhanced immunosuppressive abilities of hypoxia-preconditioned MSCs are involved in both anti-inflammatory and antifibrotic effects. 
We demonstrated that $1 \% \mathrm{O}_{2}$ MSCs promoted the production of VEGF and that VEGF knockdown reduced the anti-fibrotic effect in IRI rats. To adapt to hypoxic conditions, the upregulation of hypoxiainducible factor is responsible for gene expression of VEGF, erythropoietin, and glucose transporter 1 [3335]. Several studies have reported that VEGF functions as a renoprotective humoral factor [36-38], with evidence indicating that the administration of VEGF suppresses renal fibrosis [39]. These findings suggest that the upregulation of VEGF is implicated in the antifibrotic effect of $1 \% \mathrm{O}_{2}$ MSCs. Interestingly, we identified HGF as a downstream effector of VEGF in $1 \% \mathrm{O}_{2}$ MSCs. Previous studies have shown that HGF is a potent anti-fibrotic cytokine that antagonizes TGF- $\beta$ / Smad signaling $[40,41]$. Moreover, we previously found that a HGF-neutralizing antibody diminishes the antifibrotic effect of MSCs [31]. These results suggest that $1 \% \mathrm{O}_{2}$ MSCs enhance HGF secretion through the upregulation of VEGF, leading to their anti-fibrotic effect. In this study, we observed that HGF mRNA levels were almost equal in $1 \% \mathrm{O}_{2}$ hMSCs and $21 \% \mathrm{O}_{2}$ hMSCs. Nevertheless, $1 \% \mathrm{O}_{2}$ hMSCs showed enhanced HGF secretion. Previous studies also showed that hypoxic preconditioning enhanced HGF secretion from bone marrow-derived MSCs [24, 42]. In contrast, two recent studies reported that hypoxia-preconditioned adiposederived MSCs exhibited a reduction of such secretion $[43,44]$. These differences may be attributable to the tissues from which the MSCs were derived.

It has been reported that MSCs are characterized by the low expression of major histocompatibility complex (MHC) class I and no expression of MHC class II. Therefore, MSCs do not activate allogeneic lymphocytes [45]. Recent studies have reported that MSCs preconditioned by several inflammatory cytokines have therapeutic effects [46-48]. However, according to other studies, some cytokines upregulate MHC expression levels in MSCs, thereby elevating their immunogenicity $[49,50]$. These findings raise the possibility that cytokine-preconditioned MSCs cause allogeneic immune rejection in transplantation. In this study, $1 \% \mathrm{O}_{2}$ MSCs did not change HLA expression. AKI often occurs within a few hours or a day, and it is impossible to prepare autologous MSCs beforehand for therapy. Therefore, we attempted to demonstrate that allogeneic hypoxia-preconditioned MSC transplantation is available for treating AKI to CKD progression. For the clinical application of human MSCs, we needed to investigate whether hypoxic preconditioning enhances the antifibrotic ability of human MSCs to the same extent as rat MSCs, so we administered not only $1 \% \mathrm{O}_{2}$ rMSCs, but also $1 \% \mathrm{O}_{2}$ hMSCs to a rat IRI model. Actually, we observed that the anti-fibrotic effect of $1 \% \mathrm{O}_{2}$ MSCs was almost equivalent in MSCs derived from rats and humans.
Moreover, rat- and human-derived MSCs administered through the abdominal aorta were both observed in the kidney at day 21 post-IRI. These results suggest that hypoxia-preconditioned human MSCs have low immunogenicity and may be a good candidate for cell therapy by allogeneic transplantation. Meanwhile, Avivar-Valderas et al. reported that patients showing preexisting immunity were prone to generating donor-specific antibodies (DSA) when they were administered with allogeneic adipose MSCs. Their results suggest that sensitization may be a significant concern when patients are receiving retreatment or multi-donor trials, although DSA generation did not correlate with MSC therapeutic efficacy [51]. Our study suggests that initial allogeneic hypoxiapreconditioned MSC transplantation has the potential to be a useful therapy for preventing the progression of AKI to CKD. However, further studies are needed to determine whether re-administration of allogeneic MSCs is available for treating AKI to CKD progression.

\section{Conclusions}

In summary, the administration of $1 \% \mathrm{O}_{2}$ MSCs significantly ameliorated renal fibrosis and inflammation in IRI rats compared with $21 \% \mathrm{O}_{2}$ MSCs. We also found that $1 \% \mathrm{O}_{2}$ MSCs confer the ability to upregulate humoral factors including VEGF, HGF, and PGE2. Among them, VEGF plays an important role in not only HGF production, but also the $1 \% \mathrm{O}_{2}$ MSC-mediated anti-fibrotic effect. Our results indicate that hypoxia-preconditioned MSCs are useful as a cell therapy by allogeneic transplantation to prevent progression of AKI to CKD.

\section{Supplementary information}

Supplementary information accompanies this paper at https://doi.org/10. 1186/s13287-020-01642-6.

Additional file 1. Only slight interstitial fibrosis occurs at 7 days post-IRI. Representative images of $\mathrm{HE}$, Masson trichrome, and Sirius red staining in kidney sections at 7 days post-IRI (scale bar $=100 \mu \mathrm{m}$ ).

Additional file 2. Human and rat MSCs localize in the kidney by day 21 post-IRI. MSCs collected from enhanced green fluorescent protein (EGFP)expressing rats or Dil-labeled human MSCs were injected through the abdominal aorta after reperfusion. a Representative immunohistochemical staining of EGFP-positive cells (arrows) in the kidney cortex at 21 days postIRI (scale bar $=100 \mu \mathrm{m})$. b Representative images showing Dil-labeled human MSCs (arrowheads; scale bar $=100 \mu \mathrm{m}$ ). The right panel shows periodic acid-Schiff (PAS) staining in the same tissue section (scale bar $=100 \mu \mathrm{m})$.

Additional file 3. Hypoxic preconditioning does not change expression of MSC surface markers. Flow cytometry showing expression of surface markers on $21 \% \mathrm{O}_{2}$ hMSCs and $1 \% \mathrm{O}_{2}$ hMSCs.

Additional file 4. $1 \% \mathrm{O}_{2}$ hMSCs increase the expression of VEGF mRNA, but not HGF mRNA. a Graph showing the number of alive MSCs cultured in medium containing $10 \%$ FBS under normoxic conditions or $1 \% \mathrm{O}_{2}$ conditions. VEGF (b) and HGF (d) mRNA expression levels of MSCs were measured by PCR analysis. Data are means \pm S.D. ${ }^{\#} P<0.01,{ }^{*} P<0.05$ (one-way ANOVA followed by Bonferroni's post-hoc test or Student's ttest). 


\section{Abbreviations}

AKI: Acute kidney injury; CKD: Chronic kidney disease; CM: Conditioned medium; EGFP: Enhanced green fluorescent protein; EMT: Epithelialmesenchymal transition; HGF: Hepatocyte growth factor; HLA: Human leukocyte antigen; IRI: Ischemia-reperfusion injury; MHC: Major histocompatibility complex; MSC: Mesenchymal stem cells; $1 \% \mathrm{O}_{2}$ MSCs: MSCs cultured under $1 \% \mathrm{O}_{2}$ conditions; $21 \% \mathrm{O}_{2}$ MSCs: MSCs cultured under normoxic conditions; $1 \% \mathrm{O}_{2}$ hMSCs: Human MSCs cultured under $1 \% \mathrm{O}_{2}$ conditions; $1 \% \mathrm{O}_{2}$ hMSCs-CM: Conditioned medium from human $1 \% \mathrm{O}_{2} \mathrm{MSC}$; $21 \% \mathrm{O}_{2}$ hMSCs: Human MSCs cultured under normoxic conditions; $21 \% \mathrm{O}_{2}$ hMSCs-CM: Conditioned medium from human $21 \% \mathrm{O}_{2}$ MSCs; $1 \% \mathrm{O}_{2}$ rMSCs: Rat MSCs cultured under $1 \% \mathrm{O}_{2}$ conditions; $21 \% \mathrm{O}_{2}$ rMSCs: Rat MSCs cultured under normoxic conditions; NC siRNA $1 \% \mathrm{O}_{2}$ hMSCs: $1 \% \mathrm{O}_{2}$ hMSCs transfected with negative control siRNA; NC siRNA $1 \% \mathrm{O}_{2}$ hMSCCM: Conditioned medium from NC siRNA $/ 1 \% \mathrm{O}_{2}$ hMSCs; PGE2: Prostaglandin E2; SD: Sprague-Dawley; TGF: Transforming growth factor; VEGF: Vascular endothelial growth factor; VEGF siRNA $/ 1 \% \mathrm{O}_{2}$ hMSCs: $1 \% \mathrm{O}_{2}$ hMSCs transfected with VEGF siRNA; VEGF siRNA $1 \% \mathrm{O}_{2}$ hMSC-CM: Conditioned medium from VEGF siRNA $1 \% \mathrm{O}_{2}$ hMSCs

\section{Acknowledgements}

We would like to express our gratitude to Prof. Yukihito Higashi of the Department of Cardiovascular Regeneration and Medicine, Research Institute for Radiation Biology and Medicine, Hiroshima University, for his insightful advice on the experimental design. We also thank Ms. Miki Kagiya for sophisticated technical assistance with the experiments and Mitchell Arico from Edanz Group (www.edanzediting.com/ac) for editing a draft of this manuscript.

\section{Authors' contributions}

N.I. and A.N. designed the study. N.I., A.N., K.Y., S.M., R.K., Y.Y., T.I., and T.D. carried out experiments. N.I., A.N., S.D., Y.K., and T.M. analyzed the data. N.I., A.N., and S.D. drafted and revised the paper. All authors read and approved the final manuscript

\section{Funding}

This study was supported in part by JSPS KAKENHI grant number JP17K09699. A part of this work was carried out at the Analysis Center of Life Science, Natural Science Center for Basic Research and Development, Hiroshima University.

\section{Availability of data and materials}

The data that support the findings of this study are available from the corresponding author upon reasonable request.

\section{Ethics approval and consent to participate}

All experimental procedures were approved by the Institutional Animal Care and Use Committee of Hiroshima University (permit number, A16-83).

\section{Consent for publication}

Not applicable.

\section{Competing interests}

The Department of Stem Cell Biology and Medicine, Graduate School of Biomedical \& Health Sciences, Hiroshima University, is a collaborative research laboratory funded by TWOCELLS Company, Limited. Dr. Maeda is the deputy division manager of R\&D Division II, Head Office of Research and Development of TWOCELLS Company, Limited. Emeritus Prof. Kato is the vice president of TWOCELLS Company, Limited. Except for the abovementioned disclosures, all authors have declared that no conflict of interest exists.

\section{Author details}

'Department of Nephrology, Hiroshima University Hospital, 1-2-3 Kasumi, Minami-ku, Hiroshima 734-8551, Japan. ${ }^{2}$ Department of Stem Cell Biology and Medicine, Graduate School of Biomedical \& Health Sciences, Hiroshima University, 1-2-3 Kasumi, Minami-ku, Hiroshima 734-8553, Japan. ${ }^{3}$ TWOCELLS Company, Limited, 16-35 Hijiyama-honmachi, Minami-ku, Hiroshima 732-0816, Japan
Received: 1 November 2019 Revised: 12 February 2020

Accepted: 10 March 2020 Published online: 20 March 2020

\section{References}

1. James MT, Hemmelgarn BR, Tonelli M. Early recognition and prevention of chronic kidney disease. Lancet. 2010;375:1296-309.

2. Pani A, Bragg-Gresham J, Masala M, Piras D, Atzeni A, Pilia MG, et al. Prevalence of CKD and its relationship to eGFR-related genetic loci and clinical risk factors in the SardiNIA study cohort. J Am Soc Nephrol. 2014;25: 1533-44.

3. Mahmoodi BK, Matsushita K, Woodward M, Blankestijn PJ, Cirillo M, Ohkubo $\mathrm{T}$, et al. Associations of kidney disease measures with mortality and endstage renal disease in individuals with and without hypertension: a metaanalysis. Lancet. 2012;380:1649-61.

4. Anand S, Shivashankar R, Ali MK, Kondal D, Binukumar B, Montez-Rath ME, et al. Prevalence of chronic kidney disease in two major Indian cities and projections for associated cardiovascular disease. Kidney Int. 2015;88:178-85.

5. Coresh J, Selvin E, Stevens LA, Manzi J, Kusek JW, Eggers P, et al. Prevalence of chronic kidney disease in the United States. JAMA. 2007;298:2038-47.

6. Brück K, Stel VS, Gambaro G, Hallan S, Völzke H, Ärnlöv J, et al. CKD prevalence varies across the European general population. J Am Soc Nephrol. 2016:27:2135-47.

7. Xie Y, Bowe B, Mokdad AH, Xian H, Yan Y, Li T, et al. Analysis of the Global Burden of Disease study highlights the global, regional, and national trends of chronic kidney disease epidemiology from 1990 to 2016. Kidney Int. 2018:94:567-81.

8. Ishani A, Xue JL, Himmelfarb J, Eggers PW, Kimmel PL, Molitoris BA, et al. Acute kidney injury increases risk of ESRD among elderly. J Am Soc Nephrol. 2009;20:223-8

9. Chawla LS, Amdur RL, Amodeo S, Kimmel PL, Palant CE. The severity of acute kidney injury predicts progression to chronic kidney disease. Kidney Int. 2011;79:1361-9.

10. Coca SG, Singanamala S, Parikh CR. Chronic kidney disease after acute kidney injury: a systematic review and meta-analysis. Kidney Int. 2012;81:442-8.

11. Vielhauer V, Berning E, Eis V, Kretzler M, Segerer S, Strutz F, et al. CCR1 blockade reduces interstitial inflammation and fibrosis in mice with glomerulosclerosis and nephrotic syndrome. Kidney Int. 2004:66:2264-78.

12. Zeisberg M, Neilson EG. Mechanisms of tubulointerstitial fibrosis. J Am Soc Nephrol. 2010;21:1819-34.

13. Fu Y, Tang C, Cai J, Chen G, Zhang D, Dong Z, et al. Rodent models of AKICKD transition. Am J Physiol Renal Physiol. 2018:315:F1098-106.

14. Chawla LS, Kimmel PL. Acute kidney injury and chronic kidney disease: an integrated clinical syndrome. Kidney Int. 2012;82:516-24.

15. Tanaka S, Tanaka T, Nangaku M. Hypoxia as a key player in the AKI-to-CKD transition. Am J Physiol Renal Physiol. 2014;307:F1187-95.

16. Ferenbach DA, Bonventre JV. Mechanisms of maladaptive repair after AKI leading to accelerated kidney ageing and CKD. Nat Rev Nephrol. 2015;11: 264-76.

17. Lee RH, Pulin AA, Seo MJ, Kota DJ, Ylostalo J, Larson BL, et al. Intravenous hMSCs improve myocardial infarction in mice because cells embolized in lung are activated to secrete the anti-inflammatory protein TSG-6. Cell Stem Cell. 2009;5:54-63.

18. Chen J, Li Y, Wang L, Zhang Z, Lu D, Lu M, et al. Therapeutic benefit of intravenous administration of bone marrow stromal cells after cerebral ischemia in rats. Stroke. 2001:32:1005-11.

19. Karussis D, Kassis I, Kurkalli BG, Slavin S. Immunomodulation and neuroprotection with mesenchymal bone marrow stem cells (MSCs): a proposed treatment for multiple sclerosis and other neuroimmunological/ neurodegenerative diseases. J Neurol Sci. 2008:265:131-5.

20. Kopen GC, Prockop DJ, Phinney DG. Marrow stromal cells migrate throughout forebrain and cerebellum, and they differentiate into astrocytes after injection into neonatal mouse brains. Proc Natl Acad Sci. 1999;96:10711-6.

21. Jiang W, Ma A, Wang T, Han K, Liu Y, Zhang Y, et al. Intravenous transplantation of mesenchymal stem cells improves cardiac performance after acute myocardial ischemia in female rats. Transpl Int. 2006;19:570-80.

22. Monsel A, Zhu YG, Gennai S, Hao Q, Liu J, Lee JW. Cell-based therapy for acute organ injury: preclinical evidence and ongoing clinical trials using mesenchymal stem cells. Anesthesiology. 2014;121:1099-121.

23. Kusuma GD, Carthew J, Lim R, Frith JE. Effect of the microenvironment on mesenchymal stem cell paracrine signaling: opportunities to engineer the therapeutic effect. Stem Cells Dev. 2017;26:617-31. 
24. Chang CP, Chio CC, Cheong CU, Chao CM, Cheng BC, Lin MT. Hypoxic preconditioning enhances the therapeutic potential of the secretome from cultured human mesenchymal stem cells in experimental traumatic brain injury. Clin Sci (Lond). 2013;124:165-76.

25. Lee JH, Yoon YM, Lee SH. Hypoxic preconditioning promotes the bioactivities of mesenchymal stem cells via the HIF-1a-GRP78-Akt Axis. Int J Mol Sci. 2017;18:E1320.

26. Yoshida K, Nakashima A, Doi S, Ueno T, Okubo T, Kawano Kl, et al. Serumfree medium enhances the immunosuppressive and antifibrotic abilities of mesenchymal stem cells utilized in experimental renal fibrosis. Stem Cells Transl Med. 2018;7:893-905.

27. Wang JW, Qiu YR, Fu Y, Liu J, He ZJ, Huang ZT. Transplantation with hypoxia-preconditioned mesenchymal stem cells suppresses brain injury caused by cardiac arrest-induced global cerebral ischemia in rats. J Neurosci Res. 2017:95:2059-70.

28. Wang Z, Fang B, Tan Z, Zhang D, Ma H. Hypoxic preconditioning increases the protective effect of bone marrow mesenchymal stem cells on spinal cord ischemia/reperfusion injury. Mol Med Rep. 2016;13:1953-60.

29. Liu YY, Chiang CH, Hung SC, Chian CF, Tsai CL, Chen WC, et al. Hypoxiapreconditioned mesenchymal stem cells ameliorate ischemia/reperfusioninduced lung injury. PLoS One. 2017;12:e0187637.

30. Zhang W, Liu L, Huo Y, Yang Y, Wang Y. Hypoxia-pretreated human MSCs attenuate acute kidney injury through enhanced angiogenic and antioxidative capacities. Biomed Res Int. 2014;2014:462472.

31. Ueno T, Nakashima A, Doi S, Kawamoto T, Honda K, Yokoyama Y, et al. Mesenchymal stem cells ameliorate experimental peritoneal fibrosis by suppressing inflammation and inhibiting TGF- $\beta 1$ signaling. Kidney Int. 2013; 84:297-307.

32. Furuhashi K, Tsuboi N, Shimizu A, Katsuno T, Kim H, Saka Y, et al. Serumstarved adipose-derived stromal cells ameliorate crescentic GN by promoting immunoregulatory macrophages. J Am Soc Nephrol. 2013;24: 587-603.

33. Majmundar AJ, Wong WJ, Simon MC. Hypoxia-inducible factors and the response to hypoxic stress. Mol Cell. 2010;40:294-309.

34. Haase $\mathrm{VH}$. Regulation of erythropoiesis by hypoxia-inducible factors. Blood Rev. 2013;27:41-53.

35. Hayashi M, Sakata M, Takeda T, Yamamoto T, Okamoto Y, Sawada K, et al. Induction of glucose transporter 1 expression through hypoxia-inducible factor 1alpha under hypoxic conditions in trophoblast-derived cells. J Endocrinol. 2004;183:145-54.

36. Zhang Y, Nakano D, Guan Y, Hitomi H, Uemura A, Masaki T, et al. A sodiumglucose cotransporter 2 inhibitor attenuates renal capillary injury and fibrosis by a vascular endothelial growth factor-dependent pathway after renal injury in mice. Kidney Int. 2018;94:524-35.

37. Nangaku M. Chronic hypoxia and tubulointerstitial injury: a final common pathway to end-stage renal failure. J Am Soc Nephrol. 2006;17:17-25.

38. Tanaka T, Matsumoto M, Inagi R, Miyata T, Kojima I, Ohse T, et al. Induction of protective genes by cobalt ameliorates tubulointerstitial injury in the progressive Thy1 nephritis. Kidney Int. 2005;68:2714-25.

39. Kang DH, Hughes J, Mazzali M, Schreiner GF, Johnson RJ. Impaired angiogenesis in the remnant kidney model: II. Vascular endothelial growth factor administration reduces renal fibrosis and stabilizes renal function. J Am Soc Nephrol. 2001;12:1448-57.

40. Yang J, Dai C, Liu Y. A novel mechanism by which hepatocyte growth factor blocks tubular epithelial to mesenchymal transition. J Am Soc Nephrol. 2005;16:68-78.

41. Tan R, Zhang X, Yang J, Li Y, Liu Y. Molecular basis for the cell type specific induction of SnoN expression by hepatocyte growth factor. J Am Soc Nephrol. 2007;18:2340-9.

42. Zhang Z, Yang C, Shen M, Yang M, Jin Z, Ding L, et al. Autophagy mediates the beneficial effect of hypoxic preconditioning on bone marrow mesenchymal stem cells for the therapy of myocardial infarction. Stem Cell Res Ther. 2017;8:89.

43. Saad A, Zhu XY, Herrmann S, Hickson L, Tang H, Dietz AB, et al. Adiposederived mesenchymal stem cells from patients with atherosclerotic renovascular disease have increased DNA damage and reduced angiogenesis that can be modified by hypoxia. Stem Cell Res Ther. 2016;7:128.

44. Schive SW, Mirlashari MR, Hasvold G, Wang M, Josefsen D, Gullestad HP, et al. Human adipose-derived mesenchymal stem cells respond to shortterm hypoxia by secreting factors beneficial for human islets in vitro and potentiate antidiabetic effect in vivo. Cell Med. 2017;9:103-16.
45. Schu S, Nosov M, O'Flynn L, Shaw G, Treacy O, Barry F, et al. Immunogenicity of allogeneic mesenchymal stem cells. J Cell Mol Med. 2012;16:2094-103.

46. Pasha Z, Wang Y, Sheikh R, Zhang D, Zhao T, Ashraf M. Preconditioning enhances cell survival and differentiation of stem cells during transplantation in infarcted myocardium. Cardiovasc Res. 2008;77:134-42.

47. Herrmann JL, Wang Y, Abarbanell AM, Weil BR, Tan J, Meldrum DR. Preconditioning mesenchymal stem cells with transforming growth factoralpha improves mesenchymal stem cell-mediated cardioprotection. Shock. 2010;33:24-30

48. Ferreira JR, Teixeira GQ, Santos SG, Barbosa MA, Almeida-Porada G, Gonçalves RM. Mesenchymal stromal cell secretome: influencing therapeutic potential by cellular pre-conditioning. Front Immunol. 2018:9:2837.

49. Rafei M, Birman E, Forner K, Galipeau J. Allogeneic mesenchymal stem cells for treatment of experimental autoimmune encephalomyelitis. Mol Ther. 2009;17:1799-803.

50. Montespan F, Deschaseaux F, Sensébé L, Carosella ED, Rouas-Freiss N Osteodifferentiated mesenchymal stem cells from bone marrow and adipose tissue express HLA-G and display immunomodulatory properties in HLA-mismatched settings: implications in bone repair therapy. J Immunol Res. 2014;2014:230346.

51. Avivar-Valderas A, Martín-Martín C, Ramírez C, Del Río B, Menta R, Mancheño-Corvo $P$, et al. Dissecting allo-sensitization after local administration of human allogeneic adipose mesenchymal stem cells in perianal fistulas of Crohn's disease patients. Front Immunol. 2019;10:1244.

\section{Publisher's Note}

Springer Nature remains neutral with regard to jurisdictional claims in published maps and institutional affiliations.
Ready to submit your research? Choose BMC and benefit from:

- fast, convenient online submission

- thorough peer review by experienced researchers in your field

- rapid publication on acceptance

- support for research data, including large and complex data types

- gold Open Access which fosters wider collaboration and increased citations

- maximum visibility for your research: over $100 \mathrm{M}$ website views per year

At $\mathrm{BMC}$, research is always in progress.

Learn more biomedcentral.com/submissions 